\title{
Development and Evaluation of a Hydrophilic Matrix as a Buccoadhesive System Containing Diclofenac Sodium
}

\author{
Thaiz Cristina Wypych* and Itamar Francisco Andreazza \\ Departamento de Farmácia; Universidade Federal do Paraná; Av. Prefeito Lothario Meissner, 632; 80210-170; \\ Curitiba - PR - Brasil
}

\begin{abstract}
The aim of this work was to study the development and evaluation of a hydrolphilic matrix as a buccoadhesive system containing diclofenac sodium. Eleven formulations were prepared containing the following bioadhesive polymers: hydroxylpropylmethylcellulose, polycarbophil, guar gum and xanthan gum individually and in combination. All the formulations were evaluated for the swelling index, adhesive index, and the time of adhesive and drug release profile (\%). The results showed that the formulations that presented the most swelling index were the F3 (PAA/GX) and F6 (GG/GX). The smaller index swelling was for F1 (PAA/CM) and F10 (HPCMC/CM). The F4 (PAA/HPMC) formulation presented the best adhesive index and F10 (HPMC/CM) the worst. F1 (PAA/CM) was the best matrix hydrophilic adhesive for controlled release. The hydroxylpropylmethylcellulose, guar and xanthan gum when used individually presented low adhesiveness.
\end{abstract}

Key words: Diclofenac sodium, buccoadhesive systems, guar gum, xantan gum, hydroxypropylmethyl cellulose, polycarbophil

\section{INTRODUCTION}

Several research groups have been working on the development of pharmaceutical buccoadhesives in the form of tablets, patches, multi-layered systems, disks, micro-spheres, creams and hydrogel systems, as an alternative to conventional orally administered dosage forms (Tamburic and Craig, 1996; Desai and Kumar, 2004, MohammadiSamani, Bahri-Najafi, Yousefi, 2005; Minghetti et al., 2010). Oral mucosal drug delivery offers several benefits, such as selective release of drugs at their respective binding sites, ease of administration and removal of the dosage form, low enzyme activity, reduction of first-pass metabolism in the liver as well as the ability to control the release due to its composition of hydrophilic excipients (Lara, Garcia, Panzeri, 1998; Perioli et al., 2004; Mohammadi-samani, Bahri-najafi, Yousefi, 2005; Puthli and Dixit, 2009). Ease of access to the oral cavity gives it great potential as an environment for the administration of drugs; however one of the limitations encountered is the lack of retention of the dosage form at the point of administration. Bioadhesive dosage forms should overcome this limitation (Tamburic and Craig, 1996; ElSamaligy, Yahia, Basalious, 2004; Akbari et al., 2004; Perioli et al., 2008).

Bioadhesion is a characteristic that some natural or synthetic macro-molecules present when they adhere to biological tissue, where weight,

*Author for correspondence: thaizw@ hotmail.com 
molecular conformation, crosslink density, load, ionizing properties, as well as the concentration of the polymer used, are all determining factors for the bioadhesion to occur. In general, this process involves three stages: moistening, interpenetration and muco-polymer mechanical interaction (Peppas and Mikos, 1989; Sudhakar, Kuotsu, Bandyopadhyay, 2006; Patel, Prajapat, Patel, 2007). Modified release dosage forms release their drugs gradually, maintaining their plasmatic concentrations at therapeutic levels during a modified time period. Among the various technologies available for the preparation of these orally administered solid forms, the hydrophilic matricial system is important (Pezzini, Silva, Ferraz, 2007; Teixeira 2009). Bioadhesive systems can be characterized as a hydrophilic matrix since they are dispersions of the drug in a polymer. Their main purpose is to adhere to the mucosal layer; however, they may also be active in controlling the drug release. The most suitable material is hydrogel-forming polymer that has distinctive physical and chemical properties, such as hydrophilicity, flexibility, visco-elastic properties and an adequate degree of swelling. (Tamburic and Craig, 1996; Monaco, 2000; Elsamaligy, Yahia, Basalious, 2004; Perioli et al., 2004; Sudhakar, Kuotsu, Bandyopadhyay, 2006; Patel, Prajapati, Patel, 2007).

The release of drugs from the buccoadhesive matricial systems takes place when the dosage form comes into contact with the water, causing a change of state from vitreous to malleable. Water acts as a plastifying liquid, and is retained within the polymeric chains, thus reducing the intermolecular forces of attraction and resulting in the release of the drug (Lopes, Lobo, Costa, 2005).The purpose of this study was to develop buccoadhesive systems in the form of modifiedrelease hydrophilic matrices containing diclofenac sodium combined with four hydrogel-forming polymers: hydroxypropyl methylcellulose (HPMC), polycarbophil (PAA), guar gum (GG); and xanthan gum (GX).

\section{MATERIAL AND METHODS}

\begin{abstract}
Material
Diclofenac sodium (Galena Química e Farmacêutica Ltda, Campinas, Brazil); polycarbophil (Noveon ${ }^{\circledR}$ AA1) (Deg Importadora de Produtos Químicos Ltda, São Paulo, Brazil); high-viscosity hydroxypropyl methylcellulose (Methocel ${ }^{\circledR}$ K100MPR), (Colorcon do Brasil Ltda, Cotia, Brazil); guar gum (Purifarma Distribuidora Química e Farmacêutica Ltda, São Paulo, Brazil); xanthan gum (D'altomare Química Ltda, São Paulo, Brazil); microcrystalline cellulose (Microcel $^{\circledR} 102$ - Blanver Farmoquímica Ltda, Cotia, Brazil); deionized water; sodium tribasic phosphate (Labsynth, São Paulo, Brazil); chloridic acid (Labsynth, São Paulo, Brazil).
\end{abstract}

\section{Preparation of buccoadhesive hydrophilic matrices}

The formulations (Table 1) were prepared individually in a hydraulic press (Shimadzu SSP10A), with an $8 \mathrm{~mm}$-diameter matrix and 10 kgf of compression for $5 \mathrm{~s}$.

Microcrystalline cellulose was used as an excipient for the formulations containing only one polymer. The physical parameters of diameter, thickness and mass were calculated during matrix development.

Table 1 - Composition of buccoadhesive hidrophylic matrices (mg).

\begin{tabular}{|c|c|c|c|c|c|c|}
\hline & PAA & GG & GX & HPMC & CM & DS \\
\hline F1 & 50 & & & & 50 & 50 \\
\hline F2 & 50 & 50 & & & & 50 \\
\hline F3 & 50 & & 50 & & & 50 \\
\hline F4 & 50 & & & 50 & & 50 \\
\hline F5 & & 50 & & & 50 & 50 \\
\hline F6 & & 50 & 50 & & & 50 \\
\hline F7 & & 50 & & 50 & & 50 \\
\hline F8 & & & 50 & & 50 & 50 \\
\hline F9 & & & 50 & 50 & & 50 \\
\hline F10 & & & & 50 & 50 & 50 \\
\hline F11 & & & & & 100 & 50 \\
\hline
\end{tabular}

Legend: PAA, polycarbophil; GG, guar gum; GX, xanthan gum; HPMC, hydroxypropyl methylcellulose; CM, microcrystalline cellulose; DS, diclofenac sodium. 
Swelling Index (SI) of buccoadhesive hydrophilic matrices

The Swelling Index (SI) was determined in accordance with Desai and Kumar (2004), using pH 6.8 phosphate buffer how medium and its value was calculated with Equation (1):

$$
\mathrm{SI}=\frac{\mathrm{W}_{2}-\mathrm{W}_{1}}{\mathrm{~W}_{1}}
$$

Where $\mathrm{W}_{2}$ is the weight of the matrix after each period of swelling and $\mathrm{W}_{1}$ represents the weight of the dry matrix (before beginning of test).

Evaluation of the in vitro adhesion of the buccoadhesive hydrophilic matrices

The adhesion index (AI) was determined by adapting a methodology described by Desai and Kumar (2004) (Fig. 1).

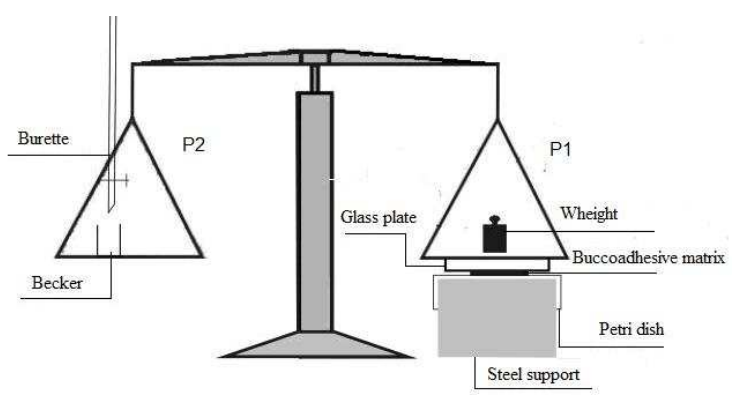

Figure 1 - Pair of scales used to evaluate the adhesion index adapted from Desai and Kumar (2004).

The buccoadhesive matrix $(\mathrm{n}=3)$ was attached with cyanoacrylate (superbonder ${ }^{\circledR}$ ) to the lower external part of the plate (P1). A Petri dish containing $2 \mathrm{~mL}$ of $\mathrm{pH} 6.8$ phosphate buffer was placed on this plate, which was supported by a stainless steel base. A $50 \mathrm{~g}$ weight was placed on the plate (P1) and kept there for 5 minutes. After the predetermined time period, water was added into the beaker positioned at $(\mathrm{P} 2)$ at a constant rate of approximately 100 droplets per minute. The addition of water was interrupted when the buccoadhesive matrix was displaced from the Petri dish. The adhesion index was expressed by the mass of water $(\mathrm{g})$ responsible for causing the displacement of the buccoadhesive matrix.

\section{In vitro adherence time of the buccoadhesive} hydrophilic matrices

The adherence time was determined using a disintegration device (Fig. 2) modified by Sudhakar, Kuotsu, Bandyopadhyay (2006).

The samples were hydrated with $50 \mu \mathrm{L}$ of $\mathrm{pH} 6.8$ phosphate buffer on one side of the matrices and fixed to a glass slide which was vertically inserted into the disintegration device. The system was activated and the time until complete erosion or detachment of the matrices from the glass slide was recorded.

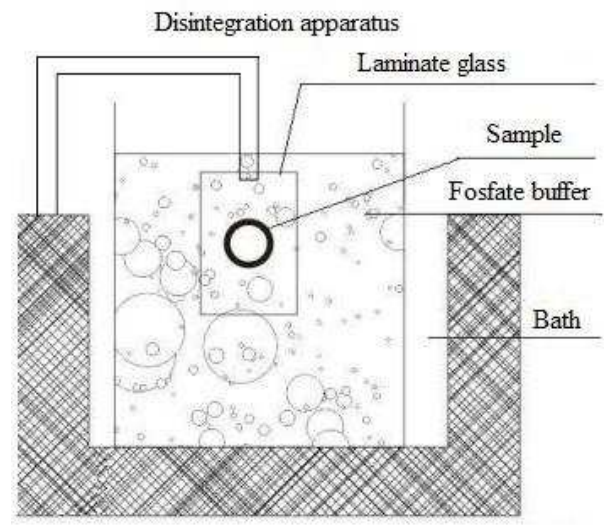

Figure 2 - Disintegration apparatus used to determination of time adherence in vitro (Sudhakar, Kuotsu, Bandyopadhyay, 2006). 
In vitro dissolution profile of the buccoadhesive hydrophilic matrices

The dissolution profile was carried out in a Hanson Research SR6 under sink conditions, for a period of 12 hours using a paddle apparatus at 50 $\mathrm{rpm}$. One of the sides of the matrix was moistened with $50 \mu \mathrm{L}$ of dissolution medium and fixed to the inside wall of a glass cube into which $500 \mathrm{~mL}$ of $\mathrm{pH} 6.8$ phosphate buffer was added as a dissolution medium. At predetermined time intervals of $5,15,30,60,90,120,180,240,300$, 360 and 720 minutes, $10 \mathrm{~mL}$ samples were withdraw, filtered (Millipore Millex-HV Hydrophilic PVDF, $0.45 \mu \mathrm{m}$ porosity) and evaluated with a spectrophotometer (UV-1601, Shimadzu $\lambda=276 \mathrm{~nm}$ ). After each sample was withdraw, the same quantity of liquid was replaced, thus maintaining the volume in the recipient.

\section{RESULTS AND DISCUSSION}

\section{Swelling Index (SI) of buccoadhesive hydrophilic matrices}

The hydrophilic matrices obtained presented an average diameter of $8 \pm 0.4 \mathrm{~mm}$; an average thickness of $2 \pm 0.1 \mathrm{~mm}$ and an average mass of $0.150 \mathrm{~g} \pm 0.007 \mathrm{~g}$.

In decreasing order, the value of the SI after 720 minutes was $\mathrm{F} 3>\mathrm{F} 6>\mathrm{F} 2>\mathrm{F} 4=\mathrm{F} 9>\mathrm{F} 7>\mathrm{F} 5>\mathrm{F} 10>\mathrm{F} 1$ (Fig. 3). Two formulations (Table 1) for the SI can not be determined, F11 because your fast disintegration (free of polymers) and the F8, which contains xanthan gum combined with microcrystalline cellulose, partially disintegrated during the first 15 minutes, and then completely 60 minutes into the test, probably due to the disintegrating effect of microcrystalline cellulose. However, when the xanthan gum was combined with the other polymers, F3 (PAA/GX) and F6 (GX/GG), the SI was elevated, proving that when combined, there is an interaction between the polymers reflected in the water absorption capacity and the integrity of the physical structure of the matrix, meaning that the latter does not disintegrate.

The partial erosion of the hydrophilic matrix, with a loss of final mass, may have been the reason for the lower SI value for F1. This occurs because the size of the matrix initially increases as the polymer hydrates and swells. After this phase, there is progressive retraction with the dissolution of the polymer and the drug until the matrix completely disappears (Lopes, Lobo, Costa, 2005).

The F10 formulation attained a low swelling index, due to the lower viscosity of HPMC, when compared to the gums and because it's concentration was too low to attain a greater rate of swelling and, consequently, gradual release of the drug. Lopes, Lobo and Costa (2005) observed that the HPMC chains, in elevated concentrations, form a tangle, giving rise to a fairly consistent gelatinous layer, which does not occur in lower concentrations, where the gel formed presents very low levels of viscosity and swelling, and the drug dissolves rapidly, so is thus not retained. The other formulations presented the expected SI values.

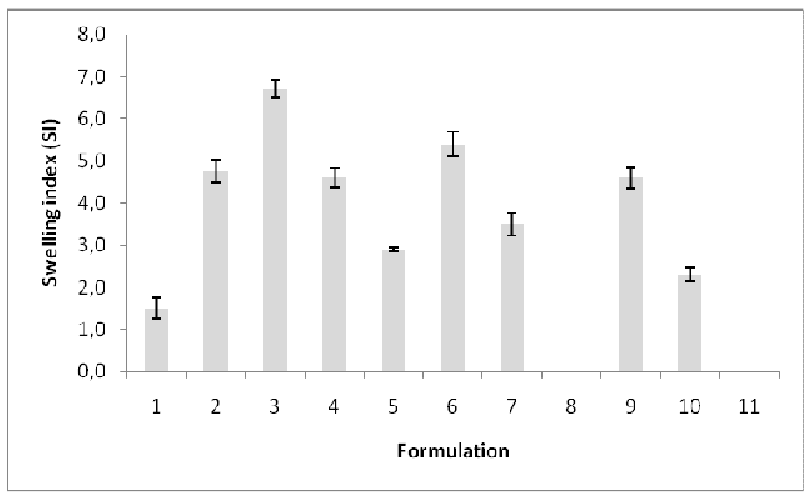

Figure 3 - Swelling index (SI) of buccoadhesive hydrophilic matrices after 720 minutes: F1,F2,F3,F4,F5,F6,F7,F8,F9,F10 e F11(n=3). 
Evaluation of the in vitro adhesion index (AI) of the buccoadhesive hydrophilic matrices

The results of the study that evaluated the AI of the buccoadhesive hydrophilic matrices are detailed in Table 2.

It can be observed that the formulations that presented better adhesion have PAA combined with other polymers in their compositions, and the best performance was achieved by F4 (PAA/HPMC). However, when these polymers are used in isolation, F1 (PAA) and F10 (HPMC), the AI is substantially reduced. Similar results were obtained by Desai and Kumar (2004) when evaluating the combination (1:1) of $\mathrm{Carbopol}^{\circledR} 934$ (a polymer derived from polyacrylic acid) and HPMC K4M, which exhibited greater adhesive power, compared to $1: 2$ and $0: 1$ ratios. When isolated, Carbopol ${ }^{\circledR} 934$ exhibited weak adhesive power.

Evaluating formulations where polymers were used in isolation, a better performance was observed for F1 (PAA), followed by F8 (GX), F5 (GG) and F10 (HPMC) (Table 2). Duchene,
Touchard and Peppas (1988) had already proved the superior adhesive performance of PAA, while guar gum presented reasonable adhesive power. In their studies, El Samaligy, Yahia and Basalious (2004) established a classification for polymers in accordance with their adhesive power, in following decreasing order: PAA $>$ GG $>$ HPMC. The results were later confirmed by Haupt and collaborators (2006).

\section{In vitro adherence time of the buccoadhesive hydrophilic matrices}

The adherence time results (Table 2) correspond to the values discovered in the adhesion study, because the formulations that attained the best AI (F4, F2 and F9) presented an adherence time greater than 24 hours, whilst the F5, F8 and F10 formulations presented lower adherence times, $0.3 \mathrm{~h}, 3 \mathrm{~h}$ and $0.2 \mathrm{~h}$ respectively. Different from other formulations, for F6 and F7, the adherence time values were not correlated with their adhesion index.

Table 2 - In vitro adhesion index and determination of adherence time of the buccoadhesive hydrophilic matrices $(\mathrm{n}=3)$.

\begin{tabular}{lcc}
\hline & Adhesion index $(\mathbf{g})$ & Adherence time $(\mathbf{h})$ \\
\hline F1 & $46,35 \pm 12,60$ & 8 \\
F2 & $130,87 \pm 14,97$ & $>24$ \\
F3 & $69,16 \pm 8,68$ & 16 \\
F4 & $151,60 \pm 10,68$ & $>24$ \\
F5 & $20,80 \pm 1,66$ & 0,3 \\
F6 & $67,82 \pm 6,96$ & $>24$ \\
F7 & $45,79 \pm 5,62$ & $>24$ \\
F8 & $28,73 \pm 1,98$ & 3 \\
F9 & $115,80 \pm 8,86$ & $>24$ \\
F10 & $17,97 \pm 1,37$ & 0,2 \\
F11 & - & - \\
\hline
\end{tabular}

\section{In vitro dissolution profile of the buccoadhesive} hydrophilic matrices

By the dissolution profile of the evaluated matrices (Fig. 4) it can be observed that F1 released around $90 \%$ of its drug in a gradual and consistent manner during the 12-hour test. The F2 profile (GG/PAA) indicates great drug retention, with about $10 \%$ dissolution after 12 hours; in other words, a reduced value compared to the formulations when these same polymers were isolated.

For F3, it was observed that drug release was low for the first 360 minutes, and after this time, the erosive process began, which facilitated drug release. The F4 (PAA/HPMC) dissolution profile was similar to that determined for F2 (GG/HPMC). Both presented the lowest rate of drug dissolution among all the formulations evaluated.

The swelling capacity of guar gum contributed to the low level of drug release, due to the formation of a layer of external gel around the F5 hydrophilic matrix. In F6, the rate of drug release in the dissolution test was observed to be low after the 12-hour test, possibly due to interaction between the gums: guar and xanthan. As a consequence of the high viscosity caused by the interaction of the two polymers, there was an increase in the layer of gel formed around the matrix, which led to the low 
dissolution value for this formulation. A synergetic effect between the guar and xanthan gums was observed by Waaler and collaborators (1992). An increase in viscosity was created when it came into contact with water, an effect that is explained by the interaction between the part containing galactomannan-free galactose and the $\alpha$-helix of xanthan gum.

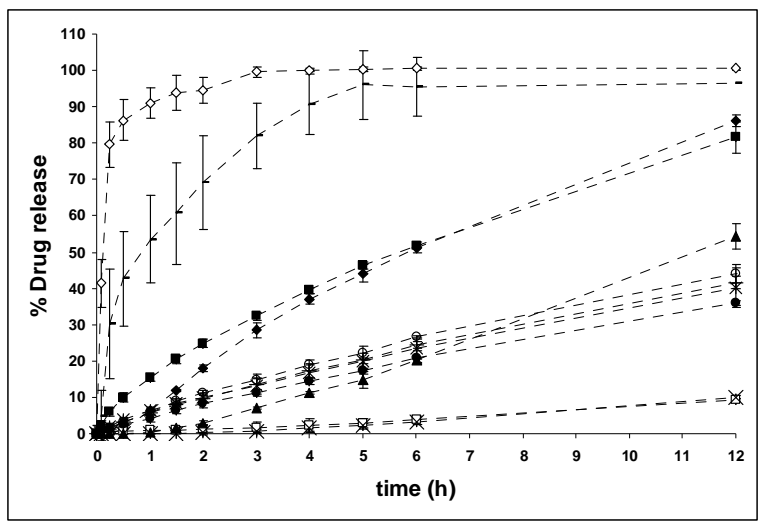

Figure 4 - Dissolution profile of the buccoadhesive hydrophilic matrix: F1--- $---;$ F2--- $\square---;$ F3---

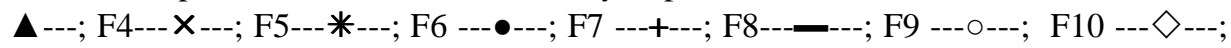
F11 ------; (n=3).

The F8 formulation disintegrated completely between 180 and 240 minutes, thus explaining its behavior during the dissolution test. In the F9 formulation, it was observed that, at the end of the dissolution test, the value obtained was approximately $44 \%$. This result matches its swelling index (4.6), a significant value when compared to the other formulations tested. F10 proved to be an immediate release dosage form. Although HPMC has the characteristic of releasing drugs in a controlled/prolonged manner, it is believed that, in this case, the concentration used was insufficient to swell the matrix and control the drug release. Compared to other polymers in this study, it was HPMC that presented one of the lowest swelling indexes.

In the F11 formulation, the quantity of microcrystalline cellulose was $100 \%$ in relation to the drug, and this high concentration may have caused a reduction in the release speed of diclofenac sodium. After 12 hours, $80 \%$ of the drug had been released, which is considered too long for an immediate release dosage form, as was predicted for F11, which contained no polymer with swelling and bioadhesive properties. Petrovick and Lima Neto (1997) state that when microcrystalline cellulose is used in concentrations greater than $80 \%$ in conjunction with low solubility drugs, it may cause a reduction in the dissolution speed of the drug.
Comparing the behavior of the polymers: PAA, HPMC, GG and GX, it was observed that when they are isolated, they present a better release performance that when they are combined. Among the matrices evaluated, those with GG presented a lower rate of drug release.

\section{Swelling index versus adhesion index}

As a rule, the greater the swelling index the greater the adhesion index. The connection between the swelling and adhesion indexes (Fig. 5) proved that the formulations that presented the greatest $\mathrm{AI}$ value (F4, F2 and F9) also attained the greatest SI value, confirming the observations of Tamburic and Craig, (1996), Prudat-Christiaens and collaborators (1996), Sudhakar, Kuotsu and Bandyopadhyay, (2006), Patel, Prajapat and Patel, (2007).

However, formulations F3 and F6 (the greatest SI values) - (Fig. 3), did not present a corresponding AI value. The excess of water absorbed by the matrix probably interfered with the adhesion process, resulting in its precocious displacement. For F10, both the adhesion index and the Swelling Index were low, evidence of the weak adhesive properties of the HPMC polymer. The F11 formulation did not present a swelling index and an adhesion index, because they did not contain any polymer in their composition. 


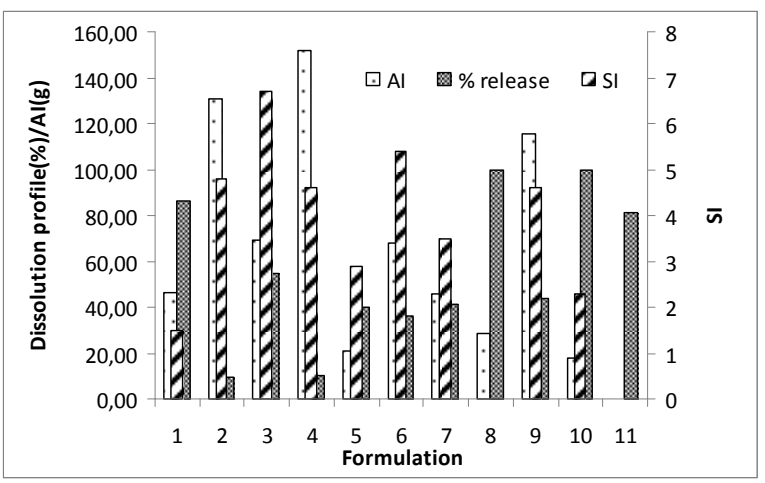

Figure 5 - Graphic exhibition of the connection between SI, AI and \% release Diclofenac sodium at the end of $720 \mathrm{~min}$.

\section{Dissolution profile versus Swelling Index}

Many authors state that the greater the swelling index of the polymer, the slower the release of the drug (Guo et al., 1998; El Samaligy, Yahia and Basalious, 2004; Lopes, Lobo, Costa, 2005; Patel, Prajapati, Patel, 2007). Due to the thick gelatinous layer formed around the exterior of the dosage form, it is more difficult for the drug to escape the polymeric network. But there are some issues that should be taken into consideration. In Figure 5, it can be observed that the F3 formulation, which attained the greatest swelling index, was not the one that presented the lowest dissolution profile. This occurred because part of the hydrophilic matrix disintegrated during the dissolution test, thus undergoing an erosive process and releasing the drug.

However, the F1, F8 and F10 formulations that presented the greatest rate of drug release were the hydrophilic matrices that presented the lowest SI, proving what several authors have observed (Guo et al.,1998; El Samaligy, Yahia and Basalious, 2004; Lopes, Lobo, Costa, 2005; Patel, Prajapati, Patel, 2007).

We work in search of a buccoadhesive matrix that met the parameters mentioned in our goal, the matrix must have adhesive capacity for a time period during which the drug dissociates to promote the desired effect. The formulation that showed the best performance was the F1, although the adhesion index was low, the adherence time was appropriate to their dissolution profile, because over the 8 hours that had been adhered, the drug had dissolution of about $60 \%$.

Then F1, F3 was the formulation that best suited to its purpose, it had a high swelling index, adherence time of 16 hours, without affecting either its dissolution profile, since after 12 hours of test there was a release of more 50\% of the drug, after 6 hours of test began the process of erosion of the matrix, the water started coming through the polymer chain, destabilizing the polymer-polymer bonds, resulting in modified-release drug.

The F3, F6, and F9 formulations, which contained xanthan gum combined with other polymers, revealed a correlation between the vales of the swelling index and the dissolution profile.

\section{CONCLUSION}

The formulations containing the combined polymers presented good adhesive power, a high swelling profile and low drug dissolution. It is supposed that this occurred due to the interaction between the chains of combined polymers, thus hindering the release of the drug. When isolated, polycarbophil (F1) presented a superior performance as a modified-release adhesive hydrophilic matrix. The matrix comprising exclusively of HPMC presented the properties of an immediate-release dosage form, with dissolution of $86 \%$ of the drug within 30 minutes, in addition to low adhesive capacity. The matrix comprising of isolated guar gum presented a profile with low drug dissolution, releasing $40 \%$ after 12 hours, probably due to the concentration of gum used compared to the quantity of drug in the formulation. Xanthan gum presented a release rate of $90 \%, 240$ minutes into the test, a low level of adhesive power and duration, and its swelling index could not be evaluated as the matrix had disintegrated during the first hour of the test. When used in an isolated manner, hydroxypropyl 
methylcellulose, guar gum and xanthan gum all presented low rates of adhesion, and are thus recommended for the preparation of dosage forms only in association with each other or with other polymers. The best association was to formulation F3, it had a high swelling index, adherence time of 16 hours, since after 12 hours of test there was a release of more $50 \%$ and presented the modifiedrelease of the drug.

\section{REFERENCES}

Akbari, J.; Nokhodchi, A.; Farid, D.; Massoud, A.; Siahi-Shadbad, M. R.; Saeedi, M. (2004), Development and evaluation of buccoadhesive propranolol hydrochloride tablet formulations: effect of fillers. Fármaco (Prat)., 5, (2), 155-161

Desai, K. G.; Kumar, T. M. (2004), Preparation and evaluation of a novel buccal adhesive system. AAPS PharmSciTech, 5, (3), 1-9

Duchêne, D.; Touchard, F.; Peppas, N. A. (1988), Pharmaceutical and medical aspects of bioadhesive systems for drug administration. Drug Dev Ind Pharm, 14, 283-318

El-Samaligy, M. S.; Yahia, S. A.; Basalious, E. B. (2004), Formulation and evaluation of diclofenac sodium buccoadhesive discs. Int. J. Pharm, 286, (1), 27-39

Guo, J. H.; Skinner, G. W.; Harcum W. W.; Barnum, P. E. (1998), Pharmaceutical applications of naturally occurring water-soluble polymers. PSTT, 1, (6), 254261

Haupt, S.; Zioni, T.; Gati, I; Kleinstern, J.; Rubinstein, A. (2006), Luminal delivery and dosing considerations of local celecoxib administration to colorectal cancer. Eur. j. pharm. sci., 28, (3), 204-211

Jug, M.; Becirevic-Lacan, M. (2004), Influence of hydroxypropyl-beta-cyclodextrin complexation on piroxicam release from buccoadhesive tablets. Eur. $j$. pharm. sci., 21, (2-3), 251-260

Khanna, R.; Agarwal, S. P.; Ahuja, A. (1996) Preparation and evaluation of bioerodible buccal tablets containing clotrimazole. Int. J. Pharm, 138, 67-73

Lara, H. G.; Garcia, A. L.; Panzeri, H. (1998), Estudos de sistemas acrílicos bioadesivos para liberação sustentada in vitro de fluoreto. Rev. Odontol. Unv. São Paulo, 12, (3), 287-291

Lima Neto, S.A., Petrovick, P.R.(1997), A celulose na farmácia. Cad. Farm., 13, (1), 19-23

Lopes, C. M.; Lobo, J. M. S.; Costa, P. (2005), Formas farmacêuticas de liberação modificada: polímeros hidrifílicos. $R B C F, \mathbf{4 1},(2), 143-154$

Minghetti, P.; Cilurzo, F.; Gennari, C. G. M.; Selmin, F.; Epstein, J. B.; Gaeta, G. M.; Colella, G. (2010), A new mucoadhesive dosage form for the management of oral lichen planus: Formulation study and clinical study. Eur J Pharm Biopharm, 1-6
Mohammadi-Samani, S.; Bahri-Najafi, R.; Yousefi, G. (2005), Formulation and in vitro evaluation of prednisolone buccoadhesive tablets. Fármaco (Prat)., 60, (4), 339-344

Monaco, J. P. (2000), Desenvolvimento de sistemas bio e mucoadesivos de uso intra-bucal:avaliação in vitro da liberação da nimesulida. Dissertação (Mestrado), Faculdade de Ciências Farmacêuticas de Ribeirão Preto, Ribeirão Preto, Brasil

Patel, V. M.; Prajapati, B. G.; Patel, M. M. (2007), Formulation, evaluation, and comparison of bilayered and multilayered mucoadhesive buccal devices of propranolol hydrochloride. AAPS PharmSciTech, 8, (1), 1-8

Peppas, N. A.; Mikos, A. G. (1989), Experimental methods for determination of bioadhesive bond strength of polymers with mucus. S.T.P. Pharma, 5, (3), 187-191

Perioli, L., Ambrogi, V.; Rubini, D.;Giovagnoli, S.; Ricci, M.; Blasi, P.; Rossi, C. (2004), Novel mucoadhesive buccal formulation containing metronidazole for the treatment of periodontal disease. J. Control. Release, 95, (3), 521-33

Perioli, L.; Pagano, C.; Mazzitelli, S.; Rossi, C.; Nastruzzi, C. (2008), Rheological and functional characterization of new antiinflammatory delivery systems designed for buccal administration. Int. J. Pharm, 356, 19-28

Pezzini, B. R.; Silva, M. A. S.; Ferraz, H.G. (2007), Formas farmacêuticas sólidas orais de liberação prolongada: sistemas monolíticos e multiparticulados. $R B C F, 43$, (4), 491-502

Prudat-Christiaens, C.; Arnaud, P.; Allain, P.; Chaumeil, J. C. (1996), Aminophylline bioadhesive tablets attempted by wet granulation. Int. J. Pharm, 141, 109-116

Puthli, S. P.; Dixit, R. P. (2009), Oral strip technology: Overview and future potential. J. Control. Release, 139, 94-107

Sudhakar, Y.; Kuotsu, K.; Bandyopadhyay A. K. (2006), Buccal bioadhesive drug delivery--a promising option for orally less efficient drugs. $J$. Control. Release, 114, (1), 15-40

Tamburic, S.; Craig Q. M. D. (1996), The Use of Bioadhesive Polymers as a Means of Improving Drug Delivery. In-Chemical Aspects of drug delivery systems, ed. The Royal Society of Chemistry, Cambridge, pp. 11-37

Teixeira, A. Z. A. (2009), Hydroxypropylcellulos Controlled Release Tablet Matrix Prepared by Wet Granulation: Effect of Powder Properties and Polymer Composition. BABT, 52, (1), 157-162

Waller, P. J.; Arnesen, K.; Graffner, C.; Muller, B.W. (1992), Optimization of the amount of xanthan gum and guar gum in a matrix tablet formulation using a central composite desing. Acta pharm. nord., 4, (4), 291-296

Received: April 13, 2010; Revised: July 01, 2010; Accepted: August 09, 2011. 